Pacific Journal of Mathematics

TIED POINTS AND STABILITY FOR A SUM OF TWO 


\title{
FIXED POINTS AND STABILITY FOR A SUM OF TWO OPERATORS IN LOCALLY CONVEX SPACES
}

\author{
G. L. Cain, JR. ANd M. Z. NAshed
}

\begin{abstract}
Some fixed point theorems for a sum of two operators are proved, generalizing to locally convex spaces a fixed point theorem of M. A. Krasnoselskii, for a sum of a completely continuous and a contraction mapping, as well as some of its recent variants.

A notion of stability of solutions of nonlinear operator equations in linear topological spaces is formulated in terms of specific topologies on the set of nonlinear operators, and a theorem on the stability of fixed points of a sum of two operators is given. As a byproduct, sufficient conditions for a mapping to be open or to be onto are obtained.
\end{abstract}

1. Introduction. Several algebraic and topological settings in the theory and applications of nonlinear operator equations lead naturally to the investigation of fixed points of a sum of two nonlinear operators, or more generally, fixed points of a mapping on the Cartesian product $X \times X$ into $X$, where $X$ is some appropriate space.

Fixed point theorems in topology and nonlinear functional analysis are usually based on certain properties (such as complete continuity, monotonicity, contractiveness, etc.) that the operator, considered as a single entity must satisfy. We recall for instance the Banach fixed point theorem, which asserts that a strict contraction on a complete metric space into itself has a unique fixed point, and the Schauder principle, which asserts that a continuous mapping $F$ on a closed convex set $K$ in a Hausdorff locally convex topological vector space $X$ into $K$ such that $F(K)$ is contained in a compact set, has a fixed point. In many problems of analysis, one encounters operators which may be split in the form $T=A+B$, where $A$ is a contraction in some sense, and $B$ is completely continuous, and $T$ itself has neither of these properties. Thus neither the Schauder fixed point theorem nor the Banach fixed point theorem applies directly in this case, and it becomes desirable to develop fixed point theorems for such situations. An early theorem of this type was given by Krasnoselskii [12]: Let $X$ be a Banach space, $S$ be a bounded closed convex subset of $X$, and $A, B$ be operators on $S$ into $X$ such that $A x+B y \in S$ for every pair $x, y \in S$. If $A$ is a strict contraction and $B$ is continuous and compact, then the equation $A x+B x=x$ has a solution in $S$. The proof of this theorem is quite simple, given the Schauder principle.

Krasnoselskii's theorem is an example of an algebraic setting which 
leads to the consideration of fixed points of a sum of two operators. In this setting, a complicated operator is split into the sum of two simpler operators which have been well investigated and for which fixed point theorems abound. For recent contributions to fixed points of this type, see Remark 3.1.

There is another setting which also leads naturally to the investigation of fixed points of a sum of two operators. This setting arises from perturbation theory. Here the operator equation $A x+B x=x$ is considered as a perturbation of $A x=x$ (or of $B x=x$ ), and one would like to assert the existence of a solution of the perturbed equation, given that the original unperturbed equation has a solution. In such a setting, there is, in general, no continuous dependence of solutions on the perturbations. If, however, one requires such continuous dependence, then we have a general problem of stability of solutions, where stability is defined in terms of certain topologies on the class of operators under consideration.

The purpose of this paper is to prove some fixed point theorems in the two settings mentioned above.

2. Definitions and preliminaries. Throughout this paper, $X$ will denote a Hausdorff locally convex topological vector space, and $\mathscr{P}$ a family of seminorms which generates the topology of $X$. For $p \in \mathscr{P}$ and $r>0$, the set $\left\{x \mid p\left(x-x_{0}\right)<r\right\}$ is denoted by $S_{p}\left(x_{0}, r\right)$. The closure of this set is denoted by $\bar{S}_{p}\left(x_{0}, r\right)$, and its boundary by $\partial S_{p}\left(x_{0}, r\right)$. We shall also sometimes use $V(p)$ to stand for $S_{p}(\theta, 1)$. A continuous mapping. $F: X \rightarrow X$ is said to be $p$-completely continuous for $p \in \mathscr{P}$ if the closure of $F\left[\bar{S}_{p}(\theta, n)\right]$ is compact for each positive integer $n$. $F$ will be called completely continuous if it is $p$-completely continuous for every $p \in \mathscr{P}$.

Several generalizations of Schauder's fixed point theorem to locally convex topological vector spaces have been made by Tychonoff [26], Hukuhara [9], Yamamuro [28], Singbal [25], Nguyen-Xuan-Loc [17], and others. In the present paper, we shall be interested in the following variants of Schauder's fixed point theorem, which are listed in order of increasing generality.

THEOREM 2.1. Let $X$ be a Hausdorff locally convex topological vector space.

(a) Let $K$ be a nonempty compact convex subset of $X$ and let $F$ be a continuous mapping of $K$ into $K$. Then $F$ has a fixed point in $K$.

(b) Let $K$ be a nonempty closed convex set in $X$ and let $F$ be a continuous mapping of $K$ into $K$ such that $F(K)$ is contained in a 
compact set. Then $F$ has a fixed point in $K$.

(c) Let $F$ be a p-completely continuous mapping of $X$ into $X$. If $F$ maps $\partial S_{p}\left(x_{0}, r\right)$ into $\bar{S}_{p}\left(x_{0}, r\right)$, then $F$ has a fixed point in $\bar{S}_{p}\left(x_{0}, r\right)$.

Part (a) is simply Tychonoff's generalization of Schauder's theorem (For a proof, see Dunford and Schwartz [4] or Bonsall [1].). A simple and interesting proof of (b) is given by Singbal [25]. Part (c) is a generalization of Rothe's version of Schauder's theorem [22].

Let $D \subset X$ and $p \in \mathscr{P}$. A mapping $A: D \rightarrow D$ is said to be a $p$-contraction if there is a $\gamma_{p}, 0 \leqq \gamma_{p}<1$, such that for all $x, y$ in $D$, $p(A x-A y) \leqq \gamma_{p} p(x-y)$.

Let $\mathscr{Q}$ be the neighborhood system of the origin obtained from $\mathscr{P}$. Thus if $U \in \mathscr{U}$, there is a finite number of seminorms $p_{1}, p_{2}, \cdots, p_{n}$ in $\mathscr{P}$ and real numbers $r_{1}, r_{2}, \cdots, r_{n}$ such that $U=\bigcap_{1}^{n} r_{i} V\left(p_{i}\right)$, where $V(p)=\{x \mid p(x)<1\}$.

THEOREM 2.2. Suppose $D$ is a sequentially complete subset of $X$ and the mapping $A: D \rightarrow D$ is a p-contraction for every $p \in \mathscr{P}$. Then $A$ has a unique fixed point $\bar{x}$ in $D$, and $A^{k} x \rightarrow \bar{x}$ for every $x \in D$.

Proof. Let $x \in D$ and let $U=\bigcap_{1}^{n} r_{i} V\left(p_{i}\right)$ be given. For any $y \in D$ and $k \geqq 1$, we have

$$
p_{i}\left(A^{k} y-y\right) \leqq\left(1-\gamma_{i}\right)^{-1} p_{i}(A y-y), \quad i=1,2, \cdots, n .
$$

Choose $m$ sufficiently large to insure that

$$
\gamma_{i}^{m}\left(1-\gamma_{i}\right)^{-1} p_{i}(A x-x) \leqq r_{i} \text { for } i=1,2, \cdots, n .
$$

Then for $y=A^{m} x$, we have

$$
\begin{aligned}
p_{i}\left(A^{m+k} x-A^{m} x\right) & \leqq\left(1-\gamma_{i}\right)^{-1} p_{i}\left(A^{m+1} x-A^{m} x\right) \\
& \leqq \gamma_{i}^{m}\left(1-\gamma_{i}\right)^{-1} p_{i}(A x-x) \leqq r_{i} .
\end{aligned}
$$

Thus $\left\{A^{k} x\right\}$ is a Cauchy sequence in $D$ and so converges to a point $\bar{x}$ in $D$. Clearly $A \bar{x}=\bar{x}$, and uniqueness of the fixed point follows as usual since $X$ is Hausdorff.

3. Fixed points of a sum of two operators in locally convex spaces. We begin with a simple theorem which generalizes Krasnoselskii's fixed point theorem [12] to locally convex spaces.

THEOREM 3.1. Let $D$ be a convex and complete subset of $X$, and $A, B$ be operators on $D$ into $X$ such that $A x+B y \in D$ for every pair $x, y \in D$. Suppose $A$ is a p-contraction for every $p \in \mathscr{P}$, and $B$ is con- 
tinuous and $B(D)$ is contained in a compact set. Then there is a point $\bar{x}$ in $D$ such that $A \bar{x}+B \bar{x}=\bar{x}$.

Proof. For each $y \in D$, the mapping $\widetilde{A}$ defined by $\widetilde{A} x=A x+B y$ is a $p$-contraction for each $p \in \mathscr{P}$ and maps $D$ into $D$, so by Theorem 2.2 , it has a fixed point, $L y$. In other words, $L y=\widetilde{A}(L y)=A(L y)+$ $B y$. Thus for all $u, v$ in $D$,

$$
L u-L v=A(L u)-A(L v)+B u-B v .
$$

So for each $p \in \mathscr{P}$, we have

$$
p(L u-L v) \leqq \gamma_{p} p(L u-L v)+p(B u-B v),
$$

or

$$
p(L u-L v) \leqq\left(1-\gamma_{p}\right)^{-1} p(B u-B v) .
$$

It is clear from (3.1) that the operator $L$ is continuous. To see that $L(D)$ is contained in a compact set, let $\left\{L x_{a}\right\}$ be a net in $L(D)$. Then $\left\{B x_{a}\right\}$ has a convergent subnet $\left\{B x_{a}^{\prime}\right\}$, since $B(D)$ is contained in a compact set. Thus $\left\{B x_{a}^{\prime}\right\}$ is a Cauchy net, and by (3.1), so also is $\left\{L x_{a}^{\prime}\right\}$. Hence $L(D)$ is contained in a compact set, so $L$ has a fixed point $\bar{x}$ in $D$, and

$$
\bar{x}=L \bar{x}=A(L \bar{x})+B \bar{x}=A \bar{x}+B \bar{x} .
$$

This completes the proof.

The various forms of the Schauder-Tychonoff theorem stated in Theorem 2.1 require a priori that a certain closed ball (or its boundary) be mapped into itself by the operator. In his work on integral equations, Dubrovskii [3] used an alternative approach of finding conditions on a completely continuous operator which guarantee the existence of some closed ball which is mapped into itself by the operator. In the next theorem, we use this technique in the setting of a sum of two operators to prove a fixed point theorem which contains as a special case a new variant of the Schauder-Tychonoff theorem in locally convex spaces. Before proceeding to the theorem, we shall give some needed definitions.

For an operator $T$, a point $x_{0} \in X$, and a real number $r>0$, define for each $p \in \mathscr{P}$,

$$
R_{p}\left(x_{0}, T, r\right)=r^{-1} \sup \left\{p\left(T x-T x_{0}\right) \mid p\left(x-x_{0}\right) \leqq r\right\}
$$

and

$$
Q_{p}\left(x_{0}, T, a\right)=\left\{r \mid R_{p}\left(x_{0}, T, r\right)<a\right\}
$$


Now consider $Q_{p}\left(x_{0}, T, a\right)$ as a subset (possibly empty) of $[0, \infty]$, the one-point compactification of $[0, \infty)$, and let $\operatorname{cl}\left(Q_{p}\left(x_{0}, T, a\right)\right)$ denote the closure of $Q_{p}\left(x_{0}, T, a\right)$ relative to $[0, \infty]$. Define

$$
\beta_{p}\left(x_{0}, T\right)=\inf \left\{a \mid \infty \in \operatorname{cl}\left(Q_{p}\left(x_{0}, T, a\right)\right)\right\} .
$$

We shall say that $T$ is $p$-quasibounded at $x_{0}$ if $\beta_{p}\left(x_{0}, T\right)$ exists. $T$ is called quasibounded at $x_{0}$ if it is $p$-quasibounded at $x_{0}$ for each $p \in \mathscr{P}$. Note that this notion of quasiboundedness generalizes that of Granas [8]. The following theorem generalizes Theorem 3 of Nashed and Wong [16].

THEOREM 3.2. Suppose the mapping $S$ is a p-contraction for every $p$ in $\mathscr{P}$, with contraction constants $\gamma_{p}$, and suppose the mapping $T$ is continuous and $\overline{T(X)}$ is compact. If $X$ is complete and if there is an $x_{0}$ in $X$ and $a p \in \mathscr{P}$ such that $T$ is $p$-quasibounded at $x_{0}$ and

$$
\gamma_{p}+\beta_{p}<1
$$

then $(I-S-T) x=z$ always has a solution.

Proof. Choose $a$ so that $\gamma_{p}+a<1$ and $\infty \in \operatorname{cl}\left(Q_{p}\left(x_{0}, T, a\right)\right)$. Let $u_{0}=(I-S-T) x_{0}$, and choose $c$ so that $c>p\left(z-u_{0}\right)\left[1-\left(\gamma_{p}+a\right)\right]^{-1}$, and $c \in Q_{p}\left(x_{0}, T, a\right)$. Then $R_{p}\left(x_{0}, T, c\right)<a$. Now define the set

$$
D=\left\{x \in X \mid p\left(x-x_{0}\right) \leqq c\right\} .
$$

It follows that for $x$ and $y$ in $D, S x+T y+z$ is in $D$ :

$$
\begin{aligned}
p\left(S x+T y+z-x_{0}\right) & =p\left(S x+T y+z-u_{0}-S x_{0}-T x_{0}\right) \\
& \leqq p\left(S x-S x_{0}\right)+p\left(T y+T x_{0}\right)+p\left(z-u_{0}\right) \\
& \leqq \gamma_{p} c+a c+\left[1-\left(\gamma_{p}+a\right)\right] c \leqq c .
\end{aligned}
$$

It now follows from Theorem 3.1 that there is an $\bar{x}$ in $D$ such that $S \bar{x}+T \bar{x}+z=\bar{x}$.

REMARK 3.1. For various fixed point theorems for a sum of two operators in Banach and Hilbert spaces, see Krasnoselskii et al. [13], [14], Browder [2], Edmunds [5], Fučík [6], [7], Kirk [11], Nashed and Wong [16], Petryshyn [18], [19], Sadovskii [23], and Webb [27]. In some of this previous work, the theorems are formulated for a mapping $F(x, y)$, not necessarily of the form $A x+B y$. Nadler [15] considered mappings defined on the Cartesian product of two metric spaces which are contractions in one variable or in each variable separately and proved that under certain conditions, such mappings have fixed points.

Essentially the same proof as that of Theorem 3.1 yields the following result. 
THEOREM 3.1'. Let $D$ be a convex and complete subset of $X$ and suppose $F: D \times D \rightarrow D$ is such that for each $p \in \mathscr{P}$, there is a constant $\gamma_{p}, 0 \leqq \gamma_{p}<1$, so that

$$
p(F(x, y)-F(x, z)) \leqq \gamma_{p} p(y-z)
$$

for all $y, z$ in $D$. Suppose further that $B: D \rightarrow D$ is continuous, $B(D)$ is contained in a compact set, and

$$
p(F(x, y)-F(z, y)) \leqq p(B x-B z) .
$$

Then there is a point $\bar{x} \in D$ for which $F(\bar{x}, \bar{x})=\bar{x}$.

REMARK 3.2. Examining the proof of Theorem 3.1, one sees that if $D=\bar{S}_{p}\left(x_{0}, r\right)$, and $X$ is complete, then we need only require that $B$ be $p$-completely continuous. (We invoke $2.1 \mathrm{c}$ to obtain a fixed point of the operator $L$.)

For the operators considered in this section, the equation

$$
A x+B x=x
$$

has a solution in particular when $A$ or $B$ is the zero operator. Thus equation (3.2) may be considered as a perturbed equation associated with

$$
A x=x \text {, or } B x=x .
$$

Theorems 3.1 and 3.2 state sufficient conditions under which the existence of a solution of either of the operator equations (3.3) is preserved with a perturbation of the operator in a certain class. We do not, however, have any information on how much of a change results in the solution. In particular a slight perturbation of the operator $A$ by an operator of type $B$ need not necessarily produce only a slight change in the solution. In other words, in the algebraic setting of Theorems 3.1 and 3.2, one does not necessarily have continuous dependence of solutions of $A x=x$ on perturbations of $A$ by operators of the type $B$ (or vice versa). We shall turn our attention in the next section to this question of continuous dependence of the solutions.

4. Stability of fixed points and solutions of nonlinear operator equations. In [10], Kasriel and Nashed formulated and investigated a notion of stability of solutions of some classes of nonlinear operator equations in Banach spaces in terms of specific topologies on the set of nonlinear operators, and obtained some results on the openness of cetain mappings as a byproduct. In this section, we extend these formulations in several directions and prove a theorem on the stability 
of fixed points for the sum of two operators.

Let $\mathscr{K}$ be a collection of continuous maps on $X$ whose domains are such that if $A_{0} \in \mathscr{K}, x_{0} \in$ domain of $A_{0}$, then $S_{p}\left(x_{0}, r\right) \subset$ domain of $A_{0}$ for $r$ sufficiently small. Let $\mathscr{T}$ be a topology on $\mathscr{K}$. Suppose $A_{0} \in \mathscr{K}, y_{0} \in X$ and $A_{0} x_{0}=y_{0}$.

Definition 4.1. The solution $x_{0}$ of $A_{0} u=y_{0}$ is called $p$-stable with respect to $(\mathscr{K}, \mathscr{T})$ if for each $r>0$ there exist $d>0$ and a neighborhood $\Omega$ of $A_{0}$ such that for all $y \in S_{p}\left(y_{0}, d\right)$ and $A \in \Omega$, there exists an $x \in S_{p}\left(x_{0}, r\right)$ such that $A x=y$. The solution $x_{0}$ is said to be a stable solution with respect to $(\mathscr{K}, \mathscr{T})$ if it is a $p$-stable solution for every $p \in \mathscr{P}$.

For $A \in \mathscr{K},\left(x_{0}, A, r\right)$ will be called a $p$-admissible triple if $\bar{S}_{p}\left(x_{0}, r\right)$ is contained in the domain of $A$.

Let $\mathscr{K}_{p}$ be the class of all continuous maps $B$ from open subsets of $X$ into $X$ which are such that $I-B$ is $p$-completely continuous. If $\left(x_{0}, B_{0}, r\right)$ is a $p$-admissible triple and $b>0$, then $\Omega_{U}\left(x_{0}, B_{0}, r, p, b\right)$ will denote the collection of all $B \in \mathscr{K}_{p}$ such that $\left(x_{0}, B, r\right)$ is a $p$-admissible triple and $p\left(B x-B_{0} x\right) \leqq b$ for all $\bar{x} \in \bar{S}_{p}\left(x_{0}, r\right)$. Let $\mathscr{T}_{p}$ be the topology on $\mathscr{K}_{p}$ generated by taking the collection of all such $\Omega_{U}$ as a subbase.

Now define

$$
\widetilde{R}_{p}\left(x_{0}, T, r\right)=r^{-1} \sup \left\{p\left(T x-T x_{0}\right) \mid p\left(x-x_{0}\right)=r\right\},
$$

and

$$
\eta_{p}\left(x_{0}, T\right)=\inf \left\{r \mid \widetilde{R}_{p}\left(x_{0}, T, r\right)<1\right\} .
$$

Note that stability for the class $\mathscr{K}$ can be reduced to consideration of equations of the form $A_{0} x=\theta$.

Theorem 4.1. Let $B_{0} \in \mathscr{K}_{p}$ and suppose $B_{0} x_{0}=\theta$. If $\eta_{p}\left(x_{0}, I-B_{0}\right)=$ 0 , then $x_{0}$ is a p-stable solution of $B_{0} x=\theta$ with respect to $\left(\mathscr{K}_{p}, \mathscr{T}_{p}\right)$.

Proof. Let $e>0$ be given. There is an $r, 0<r<e$, such that $R=\widetilde{R}_{p}\left(x_{0}, I=B_{0}, r\right)<1$. Let $a$ and $d$ be positive numbers such that $a+d<(1-R) r$. Let $B \in \Omega_{U}\left(x_{0}, B_{0}, r, p, a\right)$ and $y \in S_{p}(\theta, d)$. Consider the mapping $F$ on $\bar{S}_{p}\left(x_{0}, r\right)$ defined by $F x=x-B x+y$.

Clearly $F$ is $p$-completely continuous since $B \in \mathscr{K}_{p}$. If $F$ maps $\partial S_{p}\left(x_{0}, r\right)$ into $\bar{S}_{p}\left(x_{0}, r\right)$, it has a fixed point $\bar{x} \in \bar{S}_{p}\left(x_{0}, r\right)$ Then $B \bar{x}=y$, with $\bar{x} \in \bar{S}_{p}\left(x_{0}, r\right) \subset S_{p}\left(x_{0}, e\right)$, which proves the theorem. Now we show that $F$ indeed maps $\partial S_{p}\left(x_{0}, r\right)$ into $\bar{S}_{p}\left(x_{0}, r\right)$ :

$$
p\left(F x-x_{0}\right) \leqq p\left(x-B_{0} x-x_{0}\right)+p\left(B x-B_{0} x\right)+p(y),
$$


and

$$
p\left(x-B_{0} x-x_{0}\right) \leqq \widetilde{R}_{p}\left(x_{0}, I-B_{0}, r\right) r=R r
$$

Hence

$$
p\left(F x-x_{0}\right) \leqq R r+a+d \leqq R r+r-R r=r .
$$

If $\mathscr{K}_{0}$ is the class of all continuous operators $B$ from open subsets of $X$ into $X$ which are such that $I-B$ is completely continuous, and if $\mathscr{T}_{C}$ is the topology on $\mathscr{K}_{C}$ generated by taking as a subbase the sets $\Omega_{U}\left(x_{0}, B_{0}, r, p, b\right)$ for all $p \in \mathscr{P}$, then we have the following

CoRollaRy. If $B_{0} \in \mathscr{K}_{C}$ and $B_{0} x_{0}=\theta$, and if $\eta_{p}\left(x_{0}, I-B_{0}\right)=0$ for every $p \in \mathscr{P}$, then $x_{0}$ is a stable solution of $B_{0} x=\theta$ with respect to $\left(\mathscr{K}_{C}, \mathscr{T}_{C}\right)$.

We next turn our attention to the question of stability of sums of operators.

If $x_{0} \in X, A_{0}$ is a continuous operator, and $U \in \mathscr{Q}$, then we shall say $\left(x_{0}, A_{0}, U\right)$ is an admissible triple if $x_{0}+\bar{U} \subset$ domain $A_{0}$. (Recall that $\mathscr{U}$ is the neighborhood system of the origin obtained from $\mathscr{P}$.) Let $\mathscr{C}_{1}$ be the collection of all continuous operators $A$ which are such that $I-A$ is a $p$-contraction for every $p \in \mathscr{P}$. (Hereafter called simply a contraction.) For $A_{0}$ in $\mathscr{C}_{1}, p \in \mathscr{P}, a$ and $b$ real numbers, and $\left(x_{0}, A_{0}, U\right)$ an admissible triple, we define $\Omega_{1}\left(x_{0}, A_{0}, U, p, a, b\right)$ to be the collection of all $A$ in $\mathscr{C}_{1}$ such that

(i) $\left(x_{0}, A, U\right)$ is an admissible triple,

(ii) $p\left(\left(A-A_{0}\right) x-\left(A-A_{0}\right) x_{0}\right) \leqq b p\left(x-x_{0}\right)$ for all $x \in x_{0}+\bar{U}$,

(iii) $p\left(A x_{0}-A_{0} x_{0}\right) \leqq a$.

We define $\mathscr{T}_{1}$ to be the topology on $\mathscr{C}_{1}$ obtained by taking all such $\Omega_{1}$ as a subbase.

Let $\mathscr{C}_{2}$ be the collection of all continuous operators $B$ which are such that $I-B$ has its range contained in a compact set. For $B_{0} \in \mathscr{C}_{2}, p \in \mathscr{P}, r$ a real number, $\left(x_{0}, B_{0}, U\right)$ an admissible triple, we define $\Omega_{2}\left(x_{0}, B_{0}, U, p, r\right)$ to be the collection of all $B \in \mathscr{C}_{2}$ such that

(i) $\left(x_{0}, B, U\right)$ is an admissible triple, and

(ii) $p\left(B x-B x_{0}\right) \leqq r$ for all $x \in x_{0}+\bar{U}$.

We define $\mathscr{T}_{2}$ to be the topology on $\mathscr{C}_{2}$ with all such $\Omega_{2}$ as a subbase.

Next let $\mathscr{C}=\mathscr{C}_{1} \times \mathscr{C}_{2}$ be the Cartesian product of $\mathscr{C}_{1}$ and $\mathscr{C}_{2}$ endowed with the product topology $\mathscr{T}=\mathscr{T}_{1} \times \mathscr{T}_{2}$. Suppose $K_{0}$ is an operator such that $I-K_{0}=S_{0}+T_{0}$ for $\left(I-S_{0}, I-T_{0}\right)$ in $\mathscr{C}$.

DEFINITION 4.2. The solution $x_{0}$ of $K_{0} u=y_{0}$ is called stable with respect to $(\mathscr{C}, \mathscr{T})$ if for each $U \in \mathscr{U}$, there is a neighborhood $\Omega$ of 
$\left(I-S_{0}, I-T_{0}\right)$ and a $W \in \mathscr{C}$ such that for all $y \in y_{0}+W$ and $(I-S, I-T) \in \Omega$, there exists an $x \in x_{0}+U$ so that $K x=y$, where $I-K=S+T$.

Recall the definition of $R_{p}\left(x_{0}, T_{0}, r\right)$ and $Q_{p}\left(x_{0}, T_{0}, \alpha\right)$. For $p$ in $\mathscr{P}$ define

$$
\alpha_{p}\left(x_{0}, T_{0}\right)=\inf \left\{a \mid 0 \in \operatorname{cl}\left(Q_{p}\left(x_{0}, T_{0}, a\right)\right)\right\}
$$

THEOREM 4.2. Let $X$ be complete. Suppose $K_{0} x_{0}=y_{0}$, where $I-K_{0}=S_{0}+T_{0}$ for $\left(I-S_{0}, I-T_{0}\right)$ in $\mathscr{C}$. If $\gamma_{p}+\alpha_{p}<1$ for every $p$ in $\mathscr{P}$, then $x_{0}$ is a stable solution with respect to $(\mathscr{C}, \mathscr{T}) . \quad\left(\gamma_{p}\right.$ is $p$ contraction constant of $S_{0}$ and $\alpha_{p} \equiv \alpha_{p}\left(x_{0}, T_{0}\right)$.)

Proof. Once again we shall, without loss of generality, take $y_{0}=$ $\theta$. Let $U=\bigcap_{1}^{n} r_{i} V\left(p_{i}\right) \in \mathscr{Q}$ be given. For each $i=1,2, \cdots, n$, there is $a \xi_{i}>0$ such that $\xi_{i}+\gamma_{i}<1$ and $0 \in \operatorname{cl}\left(Q_{i}\left(x_{0}, T_{0}, \xi_{i}\right)\right)$, where $\gamma_{i}$ denotes $\gamma_{p i}$, etc. Choose $s_{i} \leqq r_{i}$ so that $R_{i}\left(x_{0}, T_{0}, s_{i}\right)<\xi_{i}$. Now choose positive constants $a_{i}, b_{i}, c_{i}, d_{i}$, for each $i=1,2, \cdots, n$, so that

$$
b_{i} s_{i}+a_{i}+2 c_{i}+d_{i}<\left(1-\xi_{i}-\gamma_{i}\right) s_{i} \text {. }
$$

Let

$$
B=I-T \in \bigcap_{1}^{n} \Omega_{2}\left(x_{0}, I-T_{0}, U, p_{i}, c_{i}\right)
$$

and

$$
A=I-S \in \bigcap_{1}^{n} \Omega_{1}\left(x_{0}, I-S_{0}, U, p_{i}, a_{i}, b_{i}\right) .
$$

Also, let $W=\bigcap_{1}^{n} d_{i} V\left(p_{i}\right)$.

Suppose $y \in W$ and consider $S x+T z+y$ for all $x$ and $z$ in $x_{0}+U^{*}$, where $U^{*}=\operatorname{cl}\left(\bigcap_{1}^{n} s_{i} V\left(p_{i}\right)\right)$. We shall show that $S x+T z+y \in x_{0}+U^{*}$ :

$$
\begin{aligned}
S x+T z+y-x_{0}= & S x+T z+y-S_{0} x_{0}-T_{0} x_{0} \\
= & \left(S x-S_{0} x_{0}\right)+\left(T z-T_{0} x_{0}\right)+y \\
= & \left(A-A_{0}\right) x-\left(A-A_{0}\right) x_{0}+S_{0} x-S_{0} x_{0} \\
& +\left(A-A_{0}\right) x_{0}+\left(T z-T_{0} z\right)+\left(T_{0} x_{0}-T_{0}\right) \\
& +\left(T_{0} z-T_{0} x_{0}\right)+y,
\end{aligned}
$$

where $A_{0}=I-S_{0}$. Now for each $i=1,2, \cdots, n$, we have

$$
\begin{aligned}
p_{i}\left(S x+T z+y-x_{0}\right) \leqq & p_{i}\left(\left(A-A_{0}\right) x-\left(A-A_{0}\right) x_{0}\right) \\
& +p_{i}\left(S_{0} x-S_{0} x_{0}\right)+p_{i}\left(\left(A-A_{0}\right) x_{0}\right) \\
& +p_{i}\left(T z-T_{0} z\right)+p_{i}\left(T_{0} x_{0}-T x_{0}\right) \\
& +p_{i}\left(T_{0} z-T_{0} x_{0}\right)+p_{i}(y) \\
\leqq & b_{i} p_{i}\left(x-x_{0}\right)+\gamma_{i} p_{i}\left(x-x_{0}\right)+a_{i}+c_{i}+c_{i} \\
& +R_{i}\left(x_{0}, T_{0}, s_{i}\right) s_{i}+p_{i}(y) \\
\leqq & \left(1-\xi_{i}-\gamma_{i}\right) s_{i}+\left(\gamma_{i}+\xi_{i}\right) s_{i}=s_{i} .
\end{aligned}
$$


So for every $x, z \in x_{0}+U^{*}$, we have $S x+T z+y \in x_{0}+U^{*}$; thus by Theorem 3.1, there is a point $\bar{x} \in x_{0}+U^{*}$ so that $S \bar{x}+T \bar{x}+y=\bar{x}$, or $K \bar{x}=y$, where $I-K=S+T$.

REMARK. If we take $T_{0}=0$ in Theorem 4.2 , we get a stability theorem for the fixed point of a contraction mapping on a complete locally convex Hausdorff topological vector space $X$. We note, however, that it is possible to formulate other notions of "contraction" for which the fixed point is not necessarily stable. Let $W_{0}$ be an open neighborhood of $\theta \in X, x_{0} \in X$, and $W=x_{0}+W_{0}$. Let $F: W \rightarrow X$. We say that $F$ is a weak contraction if there exists a convex, closed and bounded $V \subset W_{0}$ such that $x, y \in W$ and $y-x \in \lambda V$ imply $F(y)-F(x) \in \lambda \alpha V$ for some $0<\alpha<0$. Let $F$ be a weak contraction on $W$ into $X$, and $F\left(x_{0}\right)-x_{0} \in(1-\alpha) V$. Then there exists a unique fixed point $\bar{x}$ of $F$, $\bar{x} \in x_{0}+V$. However, this fixed point is obviously not necessarily stable.

5. Applications. The fixed point theorems of $\S 3$ can be applied to obtain existence theorems for mixed nonlinear integral equations of Urysohn-Volterra and Hammerstein-Volterra types in locally convex spaces in the same manner as the fixed point theorem for a sum of two operators in Banach spaces were used in [16].

We now obtain as an application of Theorem 3.1, a sufficient condition for a mapping to be open, which generalizes conditions given in [10], [20], and [21]. Recall that a mapping $F: X \rightarrow Y$ is open at a point $y_{0} \in F(X)$ if $y_{0}$ is an interior point of $F(X)$; that is, if there is a neighborhood $N$ of $y_{0}$ such that $N \subset F(X)$. It follows easily from Definition 4.2 that if $K u=y_{0}$ has a stable solution with respect to $(\mathscr{C}, \mathscr{T})$, then $K$ is open at $y_{0}$. The hypothesis of Theorem 4.2 thus also insures the openness of $K$ at $y_{0}$. We can, however, find much weaker conditions which insure that $K$ is open at $y_{0}$. To this end, define

$$
\varphi_{p}\left(x_{0}, T\right)=\inf \left\{a \mid Q_{p}\left(x_{0}, T, a\right) \neq \varnothing\right\},
$$

and suppose $K$ is as in $\S 4$; that is, $I-K=S+T$ for $(I-S, I-T)$ in $\mathscr{C}$.

THEOREM 5.1. Assume $X$ is complete. If $K x_{0}=y_{0}$ and for some $p$ in $\mathscr{P}$ it is true that $\gamma_{p}+\varphi_{p}<1$, then $K$ is open at $y_{0}$.

Proof. We may without loss of generality take $y_{0}=\theta$. Choose $\xi$ so that $Q_{p}\left(x_{0}, T, \xi\right) \neq \varnothing$ and $\gamma_{p}+\xi<1$. Let $s \in Q_{p}\left(x_{0}, T, \xi\right)$ and choose $d<\left(1-\xi-\gamma_{p}\right) s$. We shall now show that $S_{p}(\theta, d)$ is contained in the range of $K$. 
Let $u \in S_{p}(\theta, d)$ and consider $p\left(S x+T y+u-x_{0}\right)$ for $x$ and $y$ in $\bar{S}_{p}\left(x_{0}, s\right)$ :

$$
\begin{aligned}
p\left(S x+T y+u-x_{0}\right) & =p\left(S x+T y+u-S x_{0}-T x_{0}\right) \\
& \leqq p\left(S x-S x_{0}\right)+p\left(T y-T x_{0}\right)+p(u) \\
& \leqq \gamma_{p} s+\xi s+d<s .
\end{aligned}
$$

Thus by Theorem 3.1 , there is an $\bar{x} \in \bar{S}_{p}\left(x_{0}, s\right)$ such that $S \bar{x}+T \bar{x}+u=$ $\bar{x}$, which proves the theorem.

An immediate application of this result is the following theorem giving sufficient conditions for certain operators to be onto maps.

THEOREM 5.2. Let $B: X \rightarrow X$ be a continuous operator such that $T(X)$ is contained in a compact set, where $T=I-B$. Suppose for each $x \in X$, there is a $p \in \mathscr{P}$ such that $\varphi_{p}(x, T)<1$. Then the range of $B$ is $X$.

Proof. $B$ is open at each point of $B(X)$ from the previous theorem, so $B(X)$ is an open subset of $X$. We shall show that $B(X)$ is also a closed subset of $X$, and hence $B(X)$ must be all of the connected space $X$.

To show $B(X)$ is closed, let $\bar{x}$ be an accumulation point of $B(X)$ and let $\left\{y_{a}\right\}$ be a net in $B(X)$ such that $y_{a} \rightarrow \bar{x}$. Let $x_{a}$ be such that $B x_{a}=y_{a}$. Then $\left\{T x_{a}\right\}$ has a convergent subnet, say $\left\{T x_{a}^{\prime}\right\}$. Since $B x_{a}^{\prime}=$ $x_{a}^{\prime}-T x_{a}^{\prime}$, and $\left\{B x_{a}^{\prime}\right\}$ and $\left\{T x_{a}^{\prime}\right\}$ converge, we then know that $\left\{x_{a}^{\prime}\right\}$ converges. But $B x_{a}^{\prime} \rightarrow \bar{x}$, so $\bar{x} \in B(X)$. Thus $B(X)$ is closed, and the theorem is proved.

\section{REFERENCES}

1. F. E. Bonsall, Lectures on some fixed point theorems of functional analysis, Tata Institute of Fundamental Research, Bombay, India, 1962.

2. F. E. Browder, Fixed point theorems for nonlinear semicontractive mappings in Banach spaces, Arch. Rat. Mech. Anal., 21 (1966), 259-269.

3. W. Dubrovskii, Sur certaines èquations integrales nonlinéaires, Uc. Zap. Moskov Gos. Univ., 30 (1939), 49-60.

4. N. Dunford and J. T. Schwartz, Linear operators, vol. 1, Interscience Publishers Inc., New York, 1958.

5. D. E. Edmunds, Remarks on non-linear functional equations, Math. Ann., 174 (1967), 233-239.

6. S. Fučík, Fixed point theorems for a sum of nonlinear mappings, Comment. Math. Univ. Carolinae, 9 (1968), 133-143.

7. Solving of nonlinear operator equations in Banach space, Comment. Math. Univ. Carolinae, 10 (1969), 177-186.

8. A. Granas, The theory of compact vector fields and some of its applications to topology of functional spaces I, Rozpr. Matematyczne XXX (1962), 1-93.

9. M. Hukuhara, Sur l'existence des points invariants d'une transformation dans 
l'espace fonctionnel, Japanese J. Math., 20 (1950), 1-4.

10. R. H. Kasriel and M. Z. Nashed, Stability of solutions of some classes of nonlinear operator equations, Proc. Amer. Math. Soc., 17 (1966), 1036-1042.

11. W. A. Kirk, On nonlinear mappings of strongly semicontractive type, J. Math. Anal. Appl. 27 (1969), 409-412.

12. M. A. Krasnoselskii, Two remarks on the method of successive approximations, Uspeki Mat. Nauk, 10 (1955), 123-127. (Russian)

13. - and P. P. Zabreiko, Construction of a new fixed point theorem. Dokl. Akad. Nauk SSSR, 176 (1967), 1223. (Russian)

14. - , and R. I. Kachurovskii, On a fixed point theorem for operators in Hilbert spaces, Function analiz $i$ prilozen, 1, (1967), 93-94. (Russian)

15. S. Nadler, Sequences of contractions and fixed points, Pacific J. Math., 27 (1968), 579-585.

16. M. Z. Nashed, and J. S. W. Wong, Some variants of a fixed point theorem of Krasnoselskii and applications to nonlinear integral equations, J. Math. Mech., 18 (1969), 767-778.

17. Nguyen-Xuan-Loc, Fixed points and openness in a locally convex space, Proc. Amer. Math. Soc., 18 (1967), 987-991.

18. W. V. Petryshyn, Remarks on fixed point theorems and their extensions, Trans. Amer. Math. Soc., 126 (1967), 43-53.

19. - Fixed point theorems involving P-compact, semicontractive, and accretive operators not defined on all of a Banach space, J. Math. Anal. Appl., 23 (1968), 336-354. 20. M. Reichbach, Fixed points and openness, Proc. Amer. Math. Soc., 12 (1961), 734 -736 .

21. Some theorems on mappings onto, Pacific J. Math., 10 (1960), 1397-1407. 22. E. H. Rothe, Zur Theorie de Topologischen Ordunung und der Vektorfelder in Banachschen Raumen, Compositio Math., 5 (1937), 177-197.

23. B. N. Sadovskii, On a fixed point theorem, Function analiz i prilozen, 1 (1967), 7476. (Russian)

24. J. Schauder, Der Fixpunktsatz in Funktionalraumen, Studia Math., 2 (1930) 171180.

25. B. V. Singbal, Generalized form of Schauder-Tychonoff's fixed point principle, in F. E. Bonsall [1].

26. A. Tychonoff, Ein Fixpunktsatz, Math. Ann., 111 (1935), 767-776.

27. J. R. L. Webb, Fixed point theorems for nonlinear semicontractive operators in Banach spaces, J. London Math. Soc., (2) 1 (1969), 683-688.

28. S. Yamamuro, Some fixed point theorems in locally convex linear spaces, Proc. Japan Acad., 40 (1964), 1-12.

Received November 10, 1970. The work of the second author was partially supported by the U. S. Army under contract no. DA-31-124-ARO-D-462 while he was on leave from the Georgia Institute of Technology.

Georgia Institute of Technology

AND

Mathematics Research Center, University of Wisconsin 


\title{
PACIFIC JOURNAL OF MATHEMATICS
}

\author{
EDITORS
}

\author{
H. SAMELSON \\ Stanford University \\ Stanford, California 94305 \\ C. R. HOBBY \\ University of Washington \\ Seattle, Washington 98105
}

J. DugundJI

Department of Mathematics

University of Southern California

Los Angeles, California 90007

RICHARD ARENS

University of California

Los Angeles, California 90024

\section{ASSOCIATE EDITORS}
E. F. BeCKenBaCH
B. H. NeUMANN
F. WOLF
K. Yoshida

\section{SUPPORTING INSTITUTIONS}

\author{
UNIVERSITY OF BRITISH COLUMBIA \\ CALIFORNIA INSTITUTE OF TECHNOLOGY \\ UNIVERSITY OF CALIFORNIA \\ MONTANA STATE UNIVERSITY \\ UNIVERSITY OF NEVADA \\ NEW MEXICO STATE UNIVERSITY \\ OREGON STATE UNIVERSITY \\ UNIVERSITY OF OREGON \\ OSAKA UNIVERSITY
}

\author{
UNIVERSITY OF SOUTHERN CALIFORNIA \\ STANFORD UNIVERSITY \\ UNIVERSITY OF TOKYO \\ UNIVERSITY OF UTAH \\ WASHINGTON STATE UNIVERSITY \\ UNIVERSITY OF WASHINGTON \\ AMERICAN MATHEMATICAL SOCIETY \\ NAVAL WEAPONS CENTER
}

The Supporting Institutions listed above contribute to the cost of publication of this Journal, but they are not owners or publishers and have no responsibility for its content or policies.

Mathematical papers intended for publication in the Pacific Journal of Mathematics should be in typed form or offset-reproduced, (not dittoed), double spaced with large margins. Underline Greek letters in red, German in green, and script in blue. The first paragraph or two must be capable of being used separately as a synopsis of the entire paper. The editorial "we" must not be used in the synopsis, and items of the bibliography should not be cited there unless absolutely necessary, in which case they must be identified by author and Journal, rather than by item number. Manuscripts, in duplicate if possible, may be sent to any one of the four editors. Please classify according to the scheme of Math. Rev. Index to Vol. 39. All other communications to the editors should be addressed to the managing editor, Richard Arens, University of California, Los Angeles, California, 90024.

50 reprints are provided free for each article; additional copies may be obtained at cost in multiples of 50 .

The Pacific Journal of Mathematics is published monthly. Effective with Volume 16 the price per volume (3 numbers) is $\$ 8.00$; single issues, $\$ 3.00$. Special price for current issues to individual faculty members of supporting institutions and to individual members of the American Mathematical Society: $\$ 4.00$ per volume; single issues $\$ 1.50$. Back numbers are available.

Subscriptions, orders for back numbers, and changes of address should be sent to Pacific Journal of Mathematics, 103 Highland Boulevard, Berkeley, California, 94708.

PUBLISHED BY PACIFIC JOURNAL OF MATHEMATICS, A NON-PROFIT CORPORATION

Printed at Kokusai Bunken Insatsusha (International Academic Printing Co., Ltd.), 270, 3chome Totsuka-cho, Shinjuku-ku, Tokyo 160, Japan. 


\section{Pacific Journal of Mathematics}

\section{Vol. 39, No. $3 \quad$ July, 1971}

William O'Bannon Alltop, 5-designs in affine spaces ................... 547

B. G. Basmaji, Real-valued characters of metacyclic groups ................. 553

Miroslav Benda, On saturated reduced products....................... 557

J. T. Borrego, Haskell Cohen and Esmond Ernest Devun, Uniquely representable semigroups. II.......................................

George Lee Cain Jr. and Mohammed Zuhair Zaki Nashed, Fixed points and stability for a sum of two operators in locally convex spaces ....................

Donald Richard Chalice, Restrictions of Banach function spaces ...............

Eugene Frank Cornelius, Jr., A generalization of separable groups ..............

Joel L. Cunningham, Primes in products of rings ......................

Robert Alan Morris, On the Brauer group of $Z$.

593

603

615

David Earl Dobbs, Amitsur cohomology of algebraic number rings ...............

Charles F. Dunkl and Donald Edward Ramirez, Fourier-Stieltjes transforms and

weakly almost periodic functionals for compact groups ...................

Hicham Fakhoury, Structures uniformes faibles sur une classe de cônes et

d'ensembles convexes ......................................

Leslie R. Fletcher, A note on C $\theta \theta$-groups.

Humphrey Sek-Ching Fong and Louis Sucheston, On the ratio ergodic theorem for

semi-groups............................................

James Arthur Gerhard, Subdirectly irreducible idempotent semigroups ...........

Thomas Eric Hall, Orthodox semigroups.....................

Marcel Herzog, $C \theta \theta$-groups involving no Suzuki groups ..........

669

687

John Walter Hinrichsen, Concerning web-like continua ..........

691

Frank Norris Huggins, A generalization of a theorem of F. Riesz.

695

Carlos Johnson, Jr., On certain poset and semilattice homomorphisms

703

Alan Leslie Lambert, Strictly cyclic operator algebras ...........

717

Howard Wilson Lambert, Planar surfaces in knot manifolds . . .

727

Robert Allen McCoy, Groups of homeomorphisms of normed linear spaces ....... 735

T. S. Nanjundiah, Refinements of Wallis's estimate and their generalizations ...... 745

Roger David Nussbaum, A geometric approach to the fixed point index .......... 751

John Emanuel de Pillis, Convexity properties of a generalized numerical range .... 767

Donald C. Ramsey, Generating monomials for finite semigroups ....

783

William T. Reid, A disconjugacy criterion for higher order linear vector differential equations...

Roger Allen Wiegand, Modules over universal regular rings...

Kung-Wei Yang, Compact functors in categories of non-archimedean Banach

spaces.

R. Grant Woods, Correction to: "Co-absolutes of remainders of Stone-Čech compactifications".

Ronald Owen Fulp, Correction to: "Tensor and torsion products of

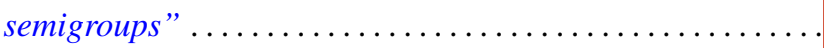

Bruce Alan Barnes, Correction to: "Banach algebras which are ideals in a banach 